\title{
Improvement of knowledge-based automatic slice-alignment method for cardiac magnetic resonance imaging
}

\author{
Shuhei Nitta ${ }^{1 *}$, Tomoyuki Takeguchi ${ }^{1}$, Nobuyuki Matsumoto ${ }^{1}$, Shigehide Kuhara ${ }^{2}$, Kenichi Yokoyama ${ }^{3}$, \\ Masamichi Imai ${ }^{3}$, Rieko Ishimura ${ }^{3}$, Toshiaki Nitatori ${ }^{3}$, Timothy Albert ${ }^{4}$
}

From 15th Annual SCMR Scientific Sessions

Orlando, FL, USA. 2-5 February 2012

\section{Background}

Automatic slice alignment allows images of the six standard cardiac planes as defined in the SCMR Image Acquisition Protocols to be obtained by simple and quick operation. Our previously reported method can detect these planes using ECG-gated breath-hold axial multislice images [1]. Achieving higher accuracy and greater robustness for variation in clinical images will lead to improved usability and reliability, resulting in easier cardiac MR examinations. To achieve these goals, we have substantially refined our previously reported automatic slice-alignment method. A combination of knowledge-based recognition and image processing techniques is applied to multiple feature point search to reduce errors in automatic detection. Volunteer and clinical data were used to evaluate of the degree of improvement.

\section{Methods}

ECG-gated 2D steady-state free precession (SSFP) axial multislice images were acquired using a 1.5-T MRI scanner (Excelart Vantage ${ }^{\mathrm{TM}}$ powered by Atlas, Toshiba Medical Systems) during a single breath-hold. The scanning conditions were TR/TE $=4.2 / 2.1$ and matrix $=$ 198x256. The slice thickness was set to $7 \mathrm{~mm}$ with no gaps, resulting in a scanning time of less than approximately $20 \mathrm{~s}$. The positions of the mitral valve, the cardiac apex, the left ventricular outflow tract, the tricuspid valve, and the right ventricular corner are detected to determine the long-axis and three short-axis orientations in order to define the 4-chamber, 2-chamber, and

${ }^{1}$ Corporate Research \& Development Center, Toshiba Corporation, Kanagawa, Japan

Full list of author information is available at the end of the article 3-chamber views using the proposed method combined with knowledge-based recognition and image processing techniques. The angular error between the results and manual annotation of the normal vector of each view was measured for three subsets (Table 1). Eighteen Japanese clinical data subsets were scored for diagnostic accuracy by two physicians (1: unacceptable, 2: marginal, but diagnostically useful, 3: good, 4: excellent).

\section{Results}

The proposed method successfully detected the six planes in 106 datasets (Table 1). The processes were completed in approximately $1.5 \mathrm{~s}(3.0-\mathrm{GHz} \mathrm{CPU})$, which is twice as fast as the conventional method. The angular error and accuracy scores are shown in Table 2. These results are more accurate than those obtained by the conventional method.

\section{Conclusions}

We have developed a sophisticated slice-alignment method employing knowledge-based recognition combined with image processing techniques to simplify cardiac scan planning. The experimental results showed that the proposed method can detect the cardiac planes more quickly and accurately than the conventional method and is more robust for data from a variety of ethnic groups.

\section{Funding}

No funding was received for this research.

\section{Author details}

'Corporate Research \& Development Center, Toshiba Corporation, Kanagawa, Japan. ${ }^{2}$ MRI Systems Division, Toshiba Medical Systems Corporation, Tochigi, 
Table 1 Number of patients and datasets.

\begin{tabular}{ccc}
\hline & patients & datasets \\
\hline Japanese healthy volunteer data & 17 & 37 \\
Japanese clinical data & 35 & 35 \\
American clinical data & 18 & 34 \\
all data & 70 & 106 \\
\hline
\end{tabular}

Table 2 The average angular errors and accuracy scores of the conventional[1] and proposed method.

\begin{tabular}{|c|c|c|c|c|c|c|}
\hline & methods & type of datasets & short-axis & 4-chamber & 2-chamber & 3-chamber \\
\hline \multirow[t]{8}{*}{ angular error[degree] } & conventional method[1] & Japanese healthy volunteer data & $3.39 \pm 1.98$ & $8.74 \pm 5.30$ & $8.81 \pm 5.74$ & $11.02 \pm 6.41$ \\
\hline & & Japanese clinical data & $5.21 \pm 7.48$ & $10.03 \pm 7.01$ & $10.92 \pm 8.94$ & $10.87 \pm 8.01$ \\
\hline & & American clinical data & $5.75 \pm 8.69$ & $9.90 \pm 8.59$ & $8.83 \pm 7.33$ & $9.55 \pm 4.94$ \\
\hline & & all data & $4.75 \pm 6.65$ & $9.54 \pm 7.01$ & $9.51 \pm 7.42$ & $10.50 \pm 6.56$ \\
\hline & proposed method & Japanese healthy volunteer data & $3.39 \pm 1.98$ & $5.12 \pm 3.44$ & $7.15 \pm 4.51$ & $5.46 \pm 3.65$ \\
\hline & & Japanese clinical data & $4.21 \pm 2.91$ & $7.79 \pm 4.10$ & $11.50 \pm 7.90$ & $6.76 \pm 4.66$ \\
\hline & & American clinical data & $4.83 \pm 3.43$ & $5.90 \pm 4.24$ & $9.64 \pm 8.25$ & $5.14 \pm 3.04$ \\
\hline & & all data & $4.12 \pm 2.85$ & $6.25 \pm 4.05$ & $9.38 \pm 7.20$ & $5.79 \pm 3.87$ \\
\hline \multirow[t]{2}{*}{ accuracy score[scale:1-4] } & conventional method[1] & Japanese clinical data(18/35) & $3.74 \pm 0.55$ & $3.47 \pm 0.69$ & $3.82 \pm 0.46$ & $3.76 \pm 0.54$ \\
\hline & proposed method & Japanese clinical data(18/35) & $3.84 \pm 0.37$ & $3.79 \pm 0.41$ & $3.82 \pm 0.39$ & $3.87 \pm 0.34$ \\
\hline
\end{tabular}

Japan. ${ }^{3}$ Department of Radiology, Kyorin University, Faculty of Medicine, Tokyo, Japan. ${ }^{4}$ Advanced Diagnostic Imaging Center, Salinas Valley Memorial Hospital, Salinas, CA, USA.

Published: 1 February 2012

\section{Reference}

1. Nitta, et al: ESMRMB. 2011, No. 726.

doi:10.1186/1532-429X-14-S1-P272

Cite this article as: Nitta et al:: Improvement of knowledge-based automatic slice-alignment method for cardiac magnetic resonance imaging. Journal of Cardiovascular Magnetic Resonance 2012 14(Suppl 1):

P272.
Submit your next manuscript to BioMed Central and take full advantage of:

- Convenient online submission

- Thorough peer review

- No space constraints or color figure charges

- Immediate publication on acceptance

- Inclusion in PubMed, CAS, Scopus and Google Scholar

- Research which is freely available for redistribution

Submit your manuscript at www.biomedcentral.com/submit
C Biomed Central 